\title{
Géolinguistique
}

14 | 2013

Varia

\section{Les parlers pygmées du bassin équatorial du Congo}

Pygmy Talks of the Congo Equatorial Basin

\section{Clothilde Chabiron, Silvia Gally et Didier Demolin}

\section{OpenEdition}

\section{Journals}

Édition électronique

URL : http://journals.openedition.org/geolinguistique/873

DOI : $10.4000 /$ geolinguistique. 873

ISSN : 2650-8176

\section{Éditeur}

UGA Éditions/Université Grenoble Alpes

\section{Édition imprimée}

Date de publication : 15 décembre 2013

Pagination : 125-144

ISBN : 978-2-84310-264-6

ISSN : 0761-9081

\section{Référence électronique}

Clothilde Chabiron, Silvia Gally et Didier Demolin, « Les parlers pygmées du bassin équatorial du Congo », Géolinguistique [En ligne], 14 | 2013, mis en ligne le 30 avril 2019, consulté le 30 octobre 2020. URL : http://journals.openedition.org/geolinguistique/873; DOI : https://doi.org/10.4000/ geolinguistique.873 


\title{
Les parlers pygmées du bassin équatorial du Congo
}

\author{
Clothilde Chabiron, Silvia Gally et Didier Demolin \\ Université Grenoble Alpes, Gipsa-lab (UMR 5216)
}

\section{Résumé}

Cet article évalue la situation linguistique des différents groupes pygmées du bassin du Congo. Ces langues se rencontrent dans la forêt qui recouvre le bassin situé sous la courbe du fleuve Congo. Leur distribution s'étend jusqu'au sud de la rivière Sankuru, dans le royaume Kuba, et à l'est vers la rivière Lomani, dans le territoire Tetela. Cette étude se concentre principalement sur quatre groupes : les Batswa qui vivent dans la partie ouest du domaine Mongo, les Batshwa qui résident parmi les Kuba, les Bafoto qui habitent dans les environs de la ville de Lisala et enfin, les Djofe qui se rencontrent dans la région d'Ikela. Les correspondances et divergences entre ces parlers sont déduites de données provenant de différentes sources : le lexique de base et quelques lexiques spécialisés des langues bantoues de la zone $\mathrm{C}$, des notes de terrain provenant de différentes sources, des informations sur la situation dialectologique de la région et une comparaison entre les lexiques des parlers pygmées avec ceux des langues parlées par leurs voisins bantous.

\section{Mots-clés}

Pygmées, Équateur, comparaison.

\section{Abstract}

This paper evaluates the linguistic situation of different Pygmy groups of the Congo basin. These languages are spoken in the forest below the curve of the Congo River and South of the Sankuru River in the Kuba territory 
and East to the Lomami River in the Tetela country. This study mainly focuses on four different groups: the Batswa living in the Western part of the Mongo domain, the Batshwa living among the Kuba, the Bafoto living close Lisala near the Congo River and the Djofe living in the region of Ikela. The affinities and differences between languages are deduced from various sources: basic and specialized lexicons of languages from the Bantu $C$ zone, fieldwork notes from various sources, data of the dialectological situation of the region and a comparison between lexicons of languages spoken by Pygmies and languages spoken by their neighbors.

\section{Keywords}

Pygmies, Equator, Comparison.

\section{Les groupes pygmées du bassin équatorial Congo}

L'identification des populations pygmées en différents groupes a fait l'objet d'un certain nombre d'études plus ou moins détaillées. Les critères de distinction les plus utilisés ont été pendant longtemps anthropologiques et géographiques et ont donné lieu à des classifications différentes. Seitz (1997) donne une classification générale en se basant sur des critères mettant en avant les aspects culturels tels que le rapport avec l'environnement écologique et les voisins ethniques, le mode d'intégration des Pygmées avec leurs voisins et la dépendance économique en fonction de leur mode de vie. Seitz (1997) situe les groupes pygmées parmi les ethnies auxquelles les différents groupes sont associés historiquement.

Il dégage deux groupes principaux pour les Pygmées de l'Équateur : les Batwa ou Batswa en contact avec les Ekonda et les Nkundo et les Batshwa qui se rencontrent chez les Kuba. À ceux-ci, il faut ajouter les Bafoto relationnés aux Ngombe, les Djofe du territoire Boyela et les Batshwa (Ato) qui se rencontrent en pays Tetela ${ }^{1}$.

1. Dans la suite de cet article, nous adoptons les conventions suivantes pour désigner les Pygmées de la région. Batswa ou Batwa pour les Pygmées vivant en contact avec les Nkundo, Ntomba et Ekonda; Bafoto pour les Pygmées relationnés aux Ngombe; Djofe pour les Pygmées en contact avec les Boyela; Batshwa pour les Pygmées qui se rencontrent chez les Kuba (Bushoong, Mbingi) et idem pour les Pygmées dans le territoire des Tetela. 


\section{Les Djofe ${ }^{2}$ et les Boyela}

Hulstaert (1986) situe les Djofe dans le bassin du fleuve Congo à l'ouest de Jwafa, autour de l'affluent Tombenga. Le lien entre les Djofe et les Boyela est établi par le fait d'union des femmes pygmées, réputées fécondes, avec les ethnies avoisinantes, plus spécifiquement les Boyela. Le Boyela fait partie du groupe Tetela (C 70), le groupe Yela, Boyela est classé C 74. L'unique publication sur le Djofe, sans comparaison avec une langue avoisinante, a été publiée par Hulstaert (1986). Hulstaert estimait, au moment où il écrivait son article, que cette langue est en voie de disparition et que la documentation disponible contient des éléments qui ne se trouvent pas dans les parlers voisins. Ceci justifiant l'importance de l'étude de cette langue à des fins comparatives. Hulstaert (1986) fait une esquisse phonologique et morphologique ainsi que des éléments de lexique.

\section{Les Batswa et les Nkundo, Ntomba, Ekonda}

Le lotswa (nom de la langue des Batswa) appartient au groupe Nkundo C 60 de la classification de Guthrie (version en ligne de la liste de référence pour la classification des langues bantoues 2009). Le groupe étant très étendu, on retrouve des Batswa dans le groupe $\mathrm{C} 61$ et $\mathrm{C}$ 61E (liés aux Ekonda). Le domaine des Batswa s'étend à l'est des rivières Ikelemba, Ruki, Busira, Momboyo et Salonga et à l'ouest autour des lacs Tumba et Mai-Ndombe, au nord jusqu'au fleuve Congo. À l'intérieur de ce territoire immense, d'autres groupes pygmées sont à cheval sur le domaine appelé Ba (Motingea, 1994), constitué des Bakote, Mbole, Ngombe, Booli, Kutshu, Saka et Ida : ce sont les Bafoto et les Balumbe (Seitz, 1997). Du point de vue de la dénomination, le nom Batswa n'est pas un terme arrêté, dans la littérature, nous trouvons aussi le nom de: Batswa, Batshua, Bachwa, Batshwa, Batua ou Batoa, le nom donné dépend de la langue voisine qui fait référence aux Pygmées (Rombauts, 1971). Les premières informations traitant des Pygmées Batswa du bassin équatorial remontent à von François (1886), qui rencontra un groupe au bord de la Tshuapa, et à Grenfell qui en rencontra un autre au bord de la Lomani (Johnston, 1908). Engels (1912) met en relief leur statut social, leur dépendance et leur mode de vie écologique. Ensuite, dans une mission entre 1920 et 1924, Van der Kerken (1944) étudie l'aspect historique, l'immigration et le développement du lien entre les tribus pygmées et leurs voisins. Au cours des années suivantes, les chercheurs et missionnaires Boelaert (1945/1947) et

2. Phonétiquement le nom de ce groupe est [jofє]. 
Hulstaert (1953) font de nombreuses études et publications en se focalisant principalement sur le rapport des Pygmées vis-à-vis des Nkundo et Ekonda. Les seuls travaux récents exclusivement portés sur le Batwa des Ekonda sont ceux d'Elshout (1963) qui traite des représentations culturelles et l'article de Muller (1964) sur une caste de chasseurs de petite taille chez les Ekonda et où il traite de la position sociale dans leur rapport aux Ekonda. Les Batwa sont souvent intégrés dans les écrits anthropologiques, mais cela apporte seulement des informations quant à l'organisation sociale par rapport à leurs voisins, ou des recherches traitant de leurs migrations dans le domaine linguistique. Les études linguistiques sont plus rares et sous forme d'ébauches. Hulstaert (1968) associe le lotswa au lokundo dans une étude comparative.

\section{Les Bafoto et les Ngombe}

Le ngombe appartient au groupe C 40 de la classification de Guthrie, plus précisément au groupe Ngombe du fleuve Congo C 41A. Les notes sur la langue des Bafoto de Hulstaert (1978) suggèrent que ces Pygmées possèdent une langue, décalée de celle de leurs voisins bantou, à la fois par le vocabulaire et par la grammaire. Cette constatation ne pourra vraiment être établie qu'à partir d'études détaillées des langues avoisinantes. Le terme Bafoto est un nom fournit par les Ngombe pour représenter ce groupe de Pygmées. Leur domaine s'étend à l'ouest de Basankusu autour de la rivière Lulonga. Hulstaert (1978) poursuit en disant que d'autres groupes de Bafoto ont été signalés dans le triangle que forment les rivières Lopori et Bolombo, mais aussi, sans donner de plus grandes précisions, plus à l'est, dans le bassin de la rivière Ikelemba. Hulstaert ajoute que cette langue, le bafoto, est, à l'époque de son article, éteinte. Son étude a été possible grâce à un vieil informateur dont le nom a été oublié, décédé avant l'achèvement de la description. Il dégage une esquisse phonologique et morphologique aussi détaillée que possible ainsi qu'un lexique. Motingea (1994) approfondi l'étude de la langue des Pygmées d'Itendo, en reprenant le travail de Hulstaert. Dans un premier chapitre, et grâce aux données récoltées, il dégage le tableau phonétique des voyelles et leurs réalisations en contexte, puis le système consonantique et ses variantes. Il travaille ensuite sur les réalisations des tons et leurs possibilités de combinaisons. Un second chapitre est dédié à la morphologie dans laquelle il dégage le système de classificateurs et les préfixes d'accord. Il extrapole ces recherches sur différents groupes pygmées, y compris en comparant la langue des Ngombe et celle des Bafoto dans les exemples. 


\section{Les Batshwa et les Bushoong, Mbingi, Kuba}

Le lotshwa appartient au groupe Bushoong C 80 de la classification de Guthrie, avec une référence plus précise au groupe Bushoong/Kuba C 83. Le territoire des Bakuba s'étend entre les rivières Kasai à l'ouest, le Sankuru au nord et la Lulua au sud-ouest (Seitz, 1997). Le territoire Kuba héberge une multitude d'ethnies dont les territoires ne sont pas délimités de façon claire. Les Batshwa vivent dispersés sur tout le territoire, mais une concentration maximum s'observe dans la partie orientale chez les Ngongo, Mbingi et Ngende. Les premiers qui mentionnent les Batshwa comme groupe à part entière sont Wolf et Wissman (1886-1887) dans un article sur «les Bacwa des Bushong». L'étude la plus complète fut ensuite réalisée en 1954 par Vansina (1954) qui s'intéressa à leurs traditions et à leur origine et par Hiernaux (1966) qui se concentra sur leurs caractéristiques anthropologiques.

\section{Éléments de cartographie}

\section{Sources et méthodologie}

La carte de la figure 1 a été réalisée par superposition des différents travaux cartographiques. Cette carte est une synthèse de tous les travaux de géolocalisation des parlers et environnements des ethnies et des groupements pygmées de cette étude. En partant d'un fond vierge (ciblé sur le bassin équatorial), nous avons retracé sur une autre planche les fleuves, rivières et villes dans un premier temps. Sur cette planche de base, nous avons ensuite pu superposer, une à une, les cartes récupérées dans la littérature, en les redimensionnant à l'échelle, afin de tracer les contours des aires des ethnies concernées par ce travail et celles de groupes pygmées. Quelles qu'elles soient, les aires sont représentatives de limites approximatives afin de situer globalement la concentration des populations. Pour les aires ethniques, c'est le travail de Hulstaert (1993), sa liste et sa carte des dialectes, ainsi que sa carte ethnique, qui ont permis de situer les groupements avec une certaine précision. Pour les aires pygmées, les informations viennent de Hutereau (1909) et de Hombert, Demolin et Philippson (2010). Les cartes de Seitz (1997) ont permis de situer précisément les groupements Batshwa des Kuba. Hulstaert (1978 et 1986) permet de situer les Bafoto et les Djofe. 


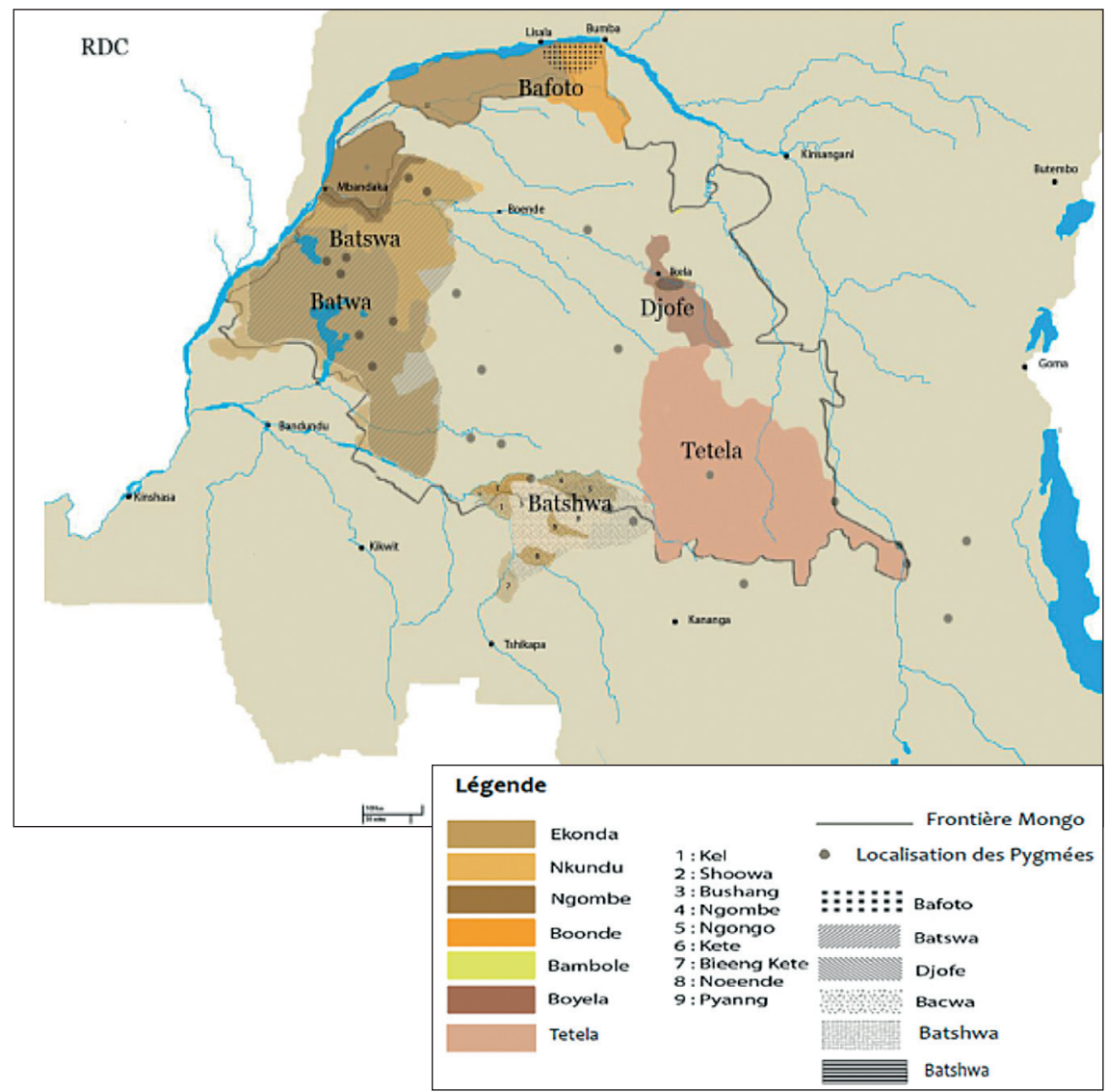

Figure 1. - Carte des aires linguistiques où se rencontrent les différents groupes pygmées de la région de l'Équateur.

Les aires linguistiques (qui correspondent aux groupes ethniques) sont représentées au moyen de plages de couleurs (plages graphiques). Les aires pygmées sont illustrées par des traits graphiques auxquels nous avons ajouté des points qui situent les groupements pygmées à partir des données de Seitz (1997).

\section{Données comparatives}

Cette partie rassemble le lexique disponible dans la littérature afin de comparer les parlers des Pygmées et des ethnies avoisinantes. Dans une première partie, la comparaison linguistique est ciblée sur les associations langue pygmée/langue voisine, puis, la deuxième partie s'articulera autour de la comparaison entre les langues pygmées. Le lexique présenté provient de diverses sources. En ce qui concerne les groupes pygmées, la plupart 
du lexique provient d'articles publiés. Certaines listes lexicales viennent de la base de données du Musée royal d'Afrique centrale de Tervuren. Le lexique est retranscrit tel qu'il est dans les ressources conformément aux choix des linguistes et/ou aux conventions de transcription.

\section{Comparaison lexicale entre Pygmées et ethnies voisines}

\section{Les Bafoto et les Ngombe}

Le vocabulaire de ces langues provient de l'ébauche de Hulstaert (1978). Les formes des mots bafoto et ngombe donnés dans le tableau sont identiques à celles qui sont présentées dans les listes de Hulstaert. Nous les avons laissées telles quelles sans essayer d'isoler les radicaux.

\begin{tabular}{|c|c|c|}
\hline Bafoto & Ngombe & Traduction \\
\hline mieka & leka & aliments \\
\hline iondo & hondo & antilope \\
\hline liekemb $\varepsilon$ & likémba & banane \\
\hline moleng $\varepsilon$ & mole & canne à sucre \\
\hline móí & móí & cœeur \\
\hline jotá & njóto & corps \\
\hline bâyع & baye & eux, elles / ceux-ci \\
\hline éko & ko & faire \\
\hline aćno & óno & ici \\
\hline yohóma & yǒma & igname \\
\hline moondá & mohóndó & jambe \\
\hline lokukú & likukú & maison / chambrette, cellule \\
\hline yehuma & ehúmá & matin \\
\hline lifembe & $f^{\prime} \cos$ & morve \\
\hline masó & mató & œufs \\
\hline loengi & mwěngi & petite promenade \\
\hline angea & ángea & placer / fixer une manche \\
\hline moísísi & mosisá & racine, tendon \\
\hline maíye & mahía & sang / pus \\
\hline sisowa & sisoa & se lever \\
\hline ndô & ndô, ndóko & seulement \\
\hline wésa & ówesa eja & tuer / faire tuer \\
\hline eka & keka & voir \\
\hline
\end{tabular}

Tableau 1. - Lexique commun bafoto / ngombe.

Les correspondances trouvées entre les lexiques bafoto et ngombe sont assez peu nombreuses dans le matériel dont nous disposons, ce qui paraît 
confirmer les impressions de Hulstaert (1978) sur les différences lexicales et grammaticales entre le bafoto et le ngombe. Parmi les différences les plus notoires, on peut noter l'effacement de la consonne h à l'initiale de certains radicaux en bafoto, comme dans le cas des désignations de l'igname : [yohóma] en bafoto $\sim$ [yǒma] en ngombe.

\section{Les Djofe et les Boyela}

Le vocabulaire de ces langues provient de l'ébauche de Hulstaert (1986) et de Molin (1933). Les formes des mots djofe et boyela données dans le tableau sont identiques à celles qui sont présentées dans les listes de Hulstaert et Molin. Nous les avons laissées telles quelles sans essayer d'isoler les radicaux.

\begin{tabular}{|l|l|l|}
\hline Boyela & Djofe & Traduction \\
\hline dikenda & $\varepsilon$ & aller \\
\hline yǒmba & omb & balayer \\
\hline ìnkòndò & kondo & banane \\
\hline dùmbù & nwa & bouche \\
\hline dó & wongo & bras \\
\hline émbá & eka & chose \\
\hline ínà & fina & danse \\
\hline băsi & aye & eau \\
\hline jàdà & zala, jala & faim \\
\hline do:do & wolo & fer \\
\hline kòdò & olo & jambe \\
\hline do:du & bele & maison \\
\hline pùdú & pulu & oiseau \\
\hline ìsó & ise & père \\
\hline yěya & $(1)$ uw(any) & savoir \\
\hline dó & lo & sommeil \\
\hline mpòmpò & umbu & vent \\
\hline yǐya & if & voler \\
\hline íyà & ph'a & voleur \\
\hline
\end{tabular}

Tableau 2. - Lexique commun djofe / boyela.

Les correspondances ne sont pas très fréquentes entre le djofe et le boyela, dans les données dont nous disposons. Cela pourrait montrer un contact récent entre les langues ou une distance géographique importante. Le principal changement dans les données est la correspondance entre l'occlusive alvéolaire sonore $d$ en boyela et l'approximante alvéolaire $l$ en djofe (parfois réalisée comme un tap $/ \mathrm{\varsigma} /$ ) : oiseau [pùdú] en boyela $\sim$ [pulu] en djofe. 


\section{Les Batswa et les Nkundo, Ntomba, Ekonda}

Les formes des mots du lotswa et du nkundo données dans le tableau sont identiques à celles qui sont présentées dans la liste de donnée de Hulstaert (1948). En ce qui concerne la langue mongo-ntomba, le lexique provient des listes lexicostatistiques (1999) du musée de Tervuren, par Ikete et Angenot (1999). Nous les avons laissées telles quelles sans essayer d'isoler les radicaux.

Les signes ['] sont repris de Hulstaert (1948) où il dit qu'ils correspondent aux nasales en nkundo. Ces sons se réalisent comme des occlusives glottales et allongent la voyelle qui précède.

\begin{tabular}{|c|c|c|c|}
\hline Lotswa & Nkundó & Ntomba & Traduction \\
\hline lonjóe & lonjwé & & abeille \\
\hline elá & kela & & afin que \\
\hline nama & nyama & & animal \\
\hline laípeé & lífé & & après demain \\
\hline olóḱ́ & olókś & & aujourd'hui \\
\hline litína & litsína & & base \\
\hline boséju & bosénju & & bois à brûler \\
\hline lokóni & lokónyi & & bois à brûler \\
\hline bonua & bomwa & bonaka & bouche \\
\hline ngua & ngua & & bouclier \\
\hline âte & wâte & & c'est-à-dire \\
\hline bovuo & bobwó & & champignon \\
\hline 'tava & ntaa & & chèvre \\
\hline bio'bà & tǒma & & choses \\
\hline 'kí'gó & nkíngó & nkingo & cou \\
\hline عpelo & cfelo & & cuisse \\
\hline kaiya & kaya (kaa) & mpaisa & donner \\
\hline loposó & lofoso & loposo & écorce \\
\hline bo'kú'du & bonkúndu & & entier \\
\hline ko & ko & & et \\
\hline la & la & & et (relie deux noms) \\
\hline lingú'já & lingánju & lokasa & feuille \\
\hline loásí & lokásá ou lokásí & & feuille de manioc \\
\hline botći & botái & & filet de chasse \\
\hline ndo'go' & ndongé & & harem \\
\hline liáná & liányá & & intelligence \\
\hline loláme & lolémi & lolemu & langue (bouche) \\
\hline 'ta'gé & ntangé & & lit \\
\hline
\end{tabular}


Suite du tableau

\begin{tabular}{|c|c|c|c|}
\hline Lotswa & Nkundó & Ntomba & Traduction \\
\hline yoóko & yoóko & & maintenant \\
\hline botú'bá & botúmbá & & maison \\
\hline livála & liála & & mariage \\
\hline njo'do & njondo & & marteau (forgeron) \\
\hline indo'gi & indenge & & martin-pêcheur \\
\hline úwa & wá (bwá) & nvei & mourir \\
\hline liíso & lǐso & liso & œil \\
\hline boelé & bokelé & bokele & œuf \\
\hline loposo & lofoso & loposo & peau \\
\hline 'kína & nkina & & peut être, ou \\
\hline embe'gá & embengá & & pigeon \\
\hline lio'ji & likonji & & piquet \\
\hline mbúla & mbúla & mbula & pluie \\
\hline 'po'ké & mpoké & & pot \\
\hline íno & íno & & presque \\
\hline te & te & & que \\
\hline mbs & mbs & & que \\
\hline 'kúma & nkúma & & quoique \\
\hline lotévú & lotćú & & rasoir \\
\hline póe & mpó & & rat \\
\hline lovébe & locmbe & & rate \\
\hline etua & etuka & & régime \\
\hline betití & betsitsí & & reins \\
\hline livále & liéle & iwele & sein \\
\hline boópu & bǒfo & wopo & semence \\
\hline njóa & njwá & & serpent \\
\hline kaika & kǐka & & seulement \\
\hline ngá & ngá & & si \\
\hline epekele & efekele & & souche (arbre) \\
\hline lokíke & lokíki & & sourcil \\
\hline lití'di & litsíndí & & talon \\
\hline lépa & léfa & & tendre (verbe) \\
\hline lóuma & lóma & & téter \\
\hline 'sa'bá & nsambá & & toit \\
\hline mó'gó & móngó & & très \\
\hline laígá & língá & & un jour \\
\hline liu’dú & likundú & ipumu & ventre \\
\hline
\end{tabular}

Tableau 3. - Correspondances lexicales batswa/nkundo, ntomba, ekonda. 
Les correspondances trouvées entre les lexiques des Pygmées batshwa, en nkundo et en ntomba montrent que ces langues sont assez proches. Les prénasalisées du nkundo et du ntomba sont réalisées comme de simples occlusives en lotswa : 'cou' [nkingo] [kigo]). Les occlusives bilabiales sourdes du lotswa subissent un processus de lénition en nkundo et en ntomba, $\mathrm{p}>\mathrm{f}$ : 'peau' [loposo] $>$ [lofoso]. Les occlusives alvéolaires sourdes du lotshwa se palatalisent devant $\mathrm{i}$ en nkundo et ntomba, $\mathrm{t}>$ ts /_i : 'reins' [betití] > [betsitsí]. Les voyelles postérieures du lotswa se semi-vocalisent en nkundo et en ntomba, u, o > w /_a : 'serpent' [njóa] > [njwá]. On note aussi quelques cas d'effacement des consonnes entre le lotswa, le nkundo et le ntomba, $\mathrm{k}>0, \mathrm{v}>0$ : 'œuf' [bokele] en nkundo, ntomba $>$ [boele] en lotswa; 'sein' [livále] en lotswa, [lićle] en nkundo, [iwele] en ntomba.

\section{Les Batshwa, les Bushoong et les Mbingi}

Le vocabulaire de ces trois langues provient des listes lexicostatistiques de Vansina archivées au musée de Tervuren. Les formes des mots tshwa (transcription cwa par Vansina) indiquées dans le tableau sont données avec les radicaux isolés des préfixes.

\begin{tabular}{|c|c|c|c|}
\hline Lotshwa & Bushoong & Mbingi & Traduction \\
\hline tí & ñtéy & fú:ngù & arbre \\
\hline bǔngí & bwiiky & ìfụllà & beaucoup \\
\hline àkèmè & yèćm & ḿbè:là & blanc \\
\hline ù-nò & nó & nú & boire \\
\hline Sèkà & Sek & òlò & bon \\
\hline ná & mun & nò & bouche \\
\hline kók-tùpyá & tuum, afú & jị̂kà & brûler \\
\hline íbù & tók & túkù & cendre \\
\hline kyàngà & ǹnóók & lú:vì & chaud \\
\hline ǹjì:lá & mbók & m̀búkà & chemin \\
\hline pụ́ & laфu & & cheveu \\
\hline m̀bwá & m̀mbwá & ḿvú & chien \\
\hline témè & ǹtém & tímà & cœur \\
\hline séké & f'ćcm, ifék & cị́kí & corne \\
\hline bóngò & kíing & pú:yù & cou \\
\hline nòdǐnò, màánò & dî́n & ńnụ, bǐnù & dent \\
\hline bilí & фéj & píy & deux \\
\hline îlwì & fonl & fímèlè & dire \\
\hline ù-pá, mbí & y'́́ḱk & pèyè & donner \\
\hline
\end{tabular}


Suite du tableau

\begin{tabular}{|c|c|c|c|}
\hline Lotshwa & Bushoong & Mbingi & Traduction \\
\hline á:ncì & màá & băfî̀, á:fị & eau \\
\hline ì: đù & yoos & pù: $\int \underline{u}$ & écorce \\
\hline ú:gwà, kǔ:ngwà & wóók & jú:kà & entendre \\
\hline é:dì & mwéżdy & wě:jị, bě:jì & étoile \\
\hline Siká & Sin & & être assis \\
\hline ịlá:lì & lakol & fí:mànà, bítàmà & être couché, dormi \\
\hline ímànà & yém't & & être debout \\
\hline ygá:tù & ǹngáát & wǔmụ̀tụ̆, bămàtà & femme \\
\hline tù-pyá & tey & yè & feu \\
\hline :cí & laa $\int$ & kíjị & feuille \\
\hline bàlè & ibady & pú:pù̀ & foie \\
\hline mpyó & pyó & m̀pyú & froid \\
\hline nì:ngà & nníín & dìngà & fumée \\
\hline bòngò & iyóon'dy & bŭngù, bàwúngú & genou \\
\hline kụ́tá & mùút & ụità & graisse \\
\hline néné & nén & ggú:ndù̀ & grand \\
\hline métù & n-núy, lún & mpàmì & homme \\
\hline némè & inc̀m & lí:mì & langue \\
\hline tálí & tady & tálì & long \\
\hline ygòn:dò & ngwoon & gò:ndò & lune \\
\hline dyà, kò- & dyá & dé & manger \\
\hline wánd & buun & kè:ndà & marcher \\
\hline kù:ncí, kưncí & nkwooc & kú:kù & montagne \\
\hline kò-fứm- & fúm & kàkà & mordre \\
\hline kwá & wa & wù & mourir \\
\hline ó:kò & bók dwèćky & ókụ̂f & nage? \\
\hline dyŭlù, myŭlù & myŏol & ú:lù & nez \\
\hline ndòmbí & ndwecm & tụ̀tù & noir \\
\hline dǐnà, mănà & díńn & nná, bịnà & nom \\
\hline nsàsà & ǹngáán & bàngò & nouveau \\
\hline bù:ngì & if $\int^{\prime} \varepsilon ́ m$ & lì:ygí & nuage \\
\hline tụ́k & butu & bǒcụ̀, bị̆tù & nuit \\
\hline dǐfò, măfò & dî́ $\int$ & ǹcú, bị::cu & œil \\
\hline kìlí & ikel & kílí & œuf \\
\hline nòní & $\phi u l$ & nfừrù̀ & oiseau \\
\hline tòlé & ito & túị & oreille \\
\hline mǐ:kà & ikody & ì:kà & os \\
\hline kóbá & iyóp & ówà & peau \\
\hline
\end{tabular}


Suite du tableau

\begin{tabular}{|c|c|c|c|}
\hline Lotshwa & Bushoong & Mbingi & Traduction \\
\hline tù & mwoot & ntù̀ & personne \\
\hline kèkè & kyéćck & nkínkè & petit \\
\hline màná & mbok & mbyé & pierre \\
\hline àyùl & yóól, tó & lù:là & plein \\
\hline cwé & mbúl & mvứlà & pluie \\
\hline Jálá & lafál & fálà & plume \\
\hline fwí & fwéy & Jí & poisson \\
\hline Sịnì & Sidy & Sijjì & pou \\
\hline é: $\int$ & ǹngél & í:là & queue \\
\hline ì:dì & mwiidy & fụ̀dị & racine \\
\hline úútú & wóot'1 & vílíngà & rond \\
\hline lèngá & twòól & ǹkùlà & rouge \\
\hline bù:ncíkí & ibúúc & ụ̀tụ̀ & sable \\
\hline à: $\int \frac{1}{1}$ & kel & kịlá & sang \\
\hline kù-ciíbà & yéé $\phi$ & bì:và, bì:pà & savoir \\
\hline ù-cú:mà & mwóóm & ứmà & $\sec$ \\
\hline bé:lè & byćcl & bé:lé & sein \\
\hline mbùtù̀ & Jáán & kúpù̀ & semence \\
\hline tá:ngwá & itááng & vì:nà & soleil \\
\hline bứ & fín, nuy & ké:cị & terre \\
\hline cwé & ǹcwey & :té & tête \\
\hline ggá & kim & ngụmmò & tous \\
\hline kù-dyàk & dyaak & & tuer \\
\hline mú:pà & m̀móc & mò & un \\
\hline ù:-lwà & $\mathrm{i}$, iy & wì & venir \\
\hline pứ & ikuun & ǹkfụ̀ndụ̀ & ventre \\
\hline nàmà & nu & dị́kà & viande \\
\hline món & món & ménà & voir \\
\hline pụ̀à & nu $\phi$ & fụ̀mbvù & voler \\
\hline
\end{tabular}

Tableau 4. - Correspondances lexicales lotswa / bushoong / mbingi.

Nous pouvons voir des correspondances entres les radicaux bushoong et tshwa : [món] 'voir' en tshwa, [món] en bushoong. Les correspondances se font généralement entre le mbingi et le tshwa. Nous trouvons aussi des cas de correspondance lexicale entre bushoong, mbingi et tshwa : [myool] 'nez' en bushoong, [u:lu] en mbingi et [myulu] en tshwa. Nous remarquons aussi que les radicaux bushoong ont tendance à perdre la voyelle finale par rapport au tshwa et au mbingi : [dîjò] 'œil' en tshwa, [diíf] en bushoong; 
[kụ́tá] 'graisse' en tshwa, [mùút] en bushoong, [ựtá] en mbingi. À partir de ces listes, nous pouvons conclure que la langue tshwa est influencée à la fois par les deux autres langues, mais l'influence du mbingi vers le tshwa est plus notable, indiquant peut-être un contact plus ancien par rapport au bushoong.

\section{Comparaison lexicale entre les différentes langues pygmées}

Le lexique présenté dans le tableau 5 prend en compte les entrées ayant un maximum de correspondances (obtenue grâce aux traductions communes des différents recueils lexicostatistique) dans toutes les langues.

\begin{tabular}{|c|c|c|c|c|}
\hline Djofe & Lotswá & Lotshwa & Bafoto & Traduction \\
\hline tange & 'ta'gé & & molenge & \\
\hline pefa & nama & & mpefa & animal \\
\hline uko & & bǔngí & & beaucoup \\
\hline nwa & bonua & ná & bonwa & bouche \\
\hline koko & bokotó & & & canne à sucre \\
\hline poa & & ǹjì:lá & & chemin \\
\hline boli & 'tava & & عmémé & chèvre \\
\hline \multirow[t]{2}{*}{ bwa } & & m̀bwá & & chien \\
\hline & 'kí'gó & bóngò & & cou \\
\hline \multirow[t]{3}{*}{ no } & & nòdǐnò, màánò & & dent \\
\hline & kaiya & ù-pá, mbí & & donner \\
\hline & loposó & yoo $\int$ & & écorce \\
\hline ona & oná & & elêa & enfant \\
\hline ngalimoto & bootsí & ygá:tù & mogálí & femme \\
\hline yowe & & tù-pyá & & feu \\
\hline ngoto & & :cí & & feuille \\
\hline tsitsi & & mpyó & & froid \\
\hline luli & & néné & & grand, grandeur \\
\hline \multirow[t]{2}{*}{ ose } & laígá & & moiho & jour \\
\hline & loláme & némè & & langue \\
\hline \multirow[t]{2}{*}{ bele } & botú'bá & & lokukú & maison \\
\hline & úwa & kwá & & mourir \\
\hline na & & dǐnà, mănà & & nom \\
\hline 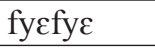 & & jònị́ & & oiseau \\
\hline woto & eo'to & & bonวwว & parent \\
\hline foso & loposo & kóbá & & peau \\
\hline ito & & tù & & personne \\
\hline kikai & & kèkè & & petit \\
\hline
\end{tabular}


Suite du tableau

\begin{tabular}{|l|l|l|l|l|}
\hline Djofe & Lotswá & Lotshwa & Bafoto & Traduction \\
\hline & 'ta'gé & mbúllà & mbesu & pluie \\
\hline wuli & & Jálá & & plume \\
\hline tsue, tshe & & Jwí & & poisson \\
\hline & & ì:dì & moísísi & racine \\
\hline & & à: ĵ̣ & maíye & sang \\
\hline & livále & bé:lè & & sein \\
\hline & boópu & mbùtù̀ & & semence \\
\hline tse & & cwé & & tête \\
\hline & & kù-dyàk & wésa & tuer \\
\hline & liu'dú & pụ́ & & ventre \\
\hline & & món & eka & voir \\
\hline
\end{tabular}

Tableau 5. - Lexique commun entre les parlers pygmées.

Les correspondances sont discutées à partir des données de ce tableau récapitulatif. Le fait qu'il ne soit pas complet pour tous les mots dans toutes les langues peut cacher certaines correspondances. Une seule correspondance est notable entre les quatre langues, elle se rapporte au mot bouche en trois réalisations tournant autour du radical [nwa], [nua], [na]. Entre le jofe et le batswa, quatre correspondances peuvent être notées : pour les mots enfant [ona], [oná]; parent [woto], [eoto]; canne à sucre [koko], [koto]; peau [foso], [poso]. Entre le djofe et le bafoto, trois correspondances : femme [ngalimoto], [mogali]; canne à sucre [tange], [molenge]; animal [pefa], [mpefa]. Entre le djofe et le tshwa, deux correspondances : chien [bwa], [mbwa]; nom [na], [nà]. Enfin, deux autres correspondances sont notables entre le lotswa et le lotshwa : mourir [uwa], [kwa] et langue [lame], [neme]. En ce qui concerne les couples bafoto/lotswa ou bafoto/ batshwa, aucune correspondance ne peut être relevée. À partir de ces données, nous pouvons émettre l'hypothèse que le djofe serait plus proche du batswa, que du lotshwa et du bafoto. Le lotswa et le lotshwa semblent aussi assez proches. La raison pour laquelle il y a peu de correspondances entre bafoto et lotshwa peut s'expliquer par une situation géographique éloignée. Le lotswa et le bafoto, géographiquement proches, paraissent pourtant aussi assez éloignés. Des données plus complètes pourraient permettre de répondre à cette question. 


\section{Correspondances avec le proto-bantou}

Le vocabulaire présenté dans le tableau 6 met en relation les correspondances les plus fréquentes entre les langues des Pygmées, celles de leurs voisins et le proto-bantou. Ces données viennent des vocabulaires disponibles sur les langues pygmées et de listes lexicostatistiques des différentes langues examinées. Les reconstructions proto-bantou viennent des «Bantu lexical reconstruction 3 » que l'on peut obtenir sur le site du Musée royal de l'Afrique centrale de Tervuren. Ce tableau met en évidence un certain nombre de correspondances et d'évolutions phonétiques connues en bantou. Le faible nombre d'entrées lexicales et le petit nombre de correspondances pour chaque langue montre qu'il est nécessaire d'avoir des données plus importantes pour mettre en évidence des tendances claires dans l'évolution entre le proto-bantou, les parlés des Pygmées et des peuples bantous du bassin du Congo. 


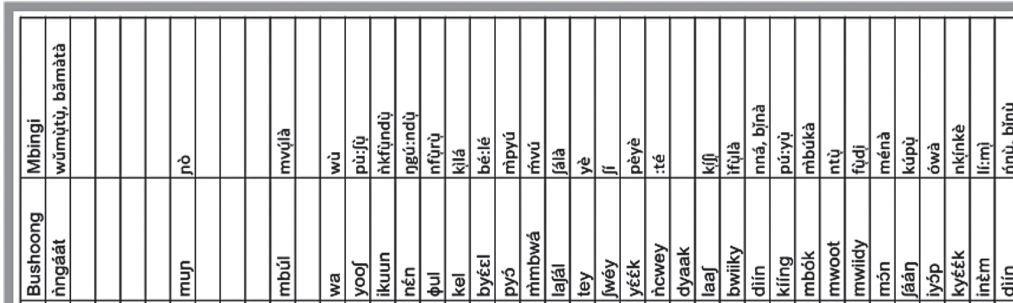

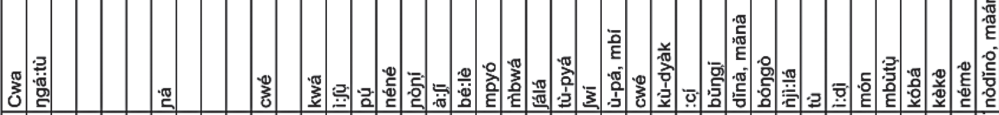

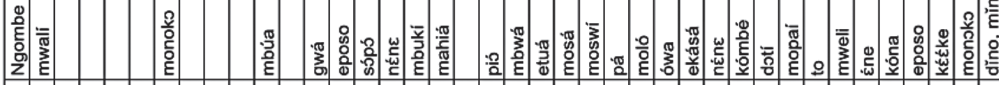

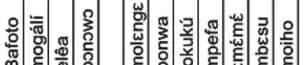

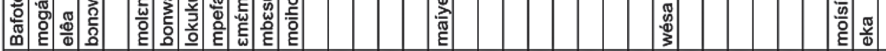

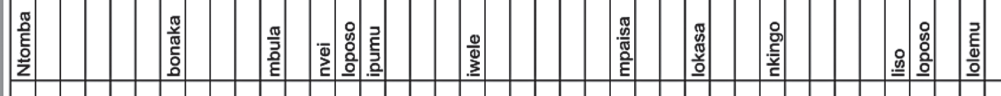

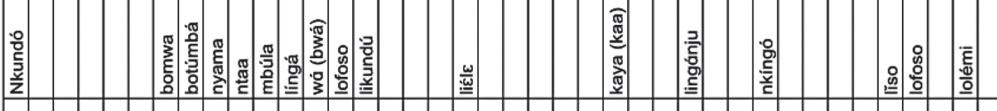

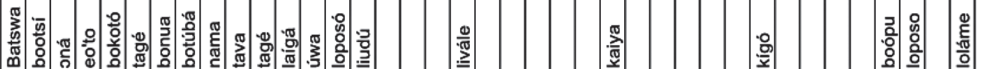

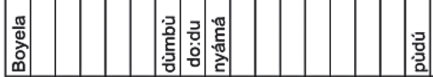

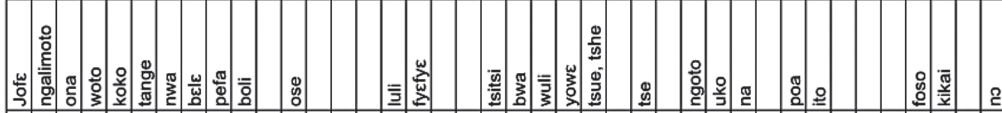

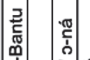

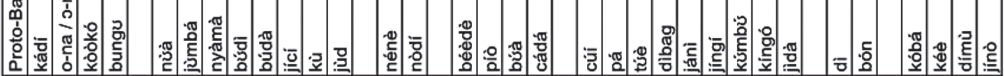

哀

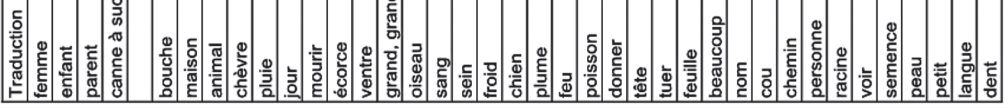




\section{RÉFÉRENCES BIBLIOGRAPHIQUES}

Boelaert Edmond, 1945, «Les Batswa de l'Équateur», Annales Aequatoria, vol. 8, $\mathrm{n}^{\circ} 1$, p. 26.

Boelaert Edmond, 1946a, «Quelques notes sur les Batswa de l'Équateur», Annale Aequatoria, vol. 9, nº 2, p. 58-63.

Boelaert Edmond, 1946b, «Les Batswa», Annales Aequatoria, vol. 9, no 4, p. 153 154.

Boelaert Edmond, 1947, «Les Batswa, quelques notes démographiques », Annales Aequatoria, vol. 10, p. 134-136.

Elshout Pierre, 1963, «Les Batwa des Ekonda», Archives d'Ethnographie, nº 6, Tervuren, Belgique.

Engels A., 1911/1912, «Notes sur les Batshua (Batwa)», Revue congolaise, p. 214-217.

FrançOIs Curt von, 1886, Uber seine Reisen im sudlichen Kongobecken (Sur ces voyages dans le bassin du Congo méridional), Verh. D. Gessel. F. Erdkunde zu Berlin, vol. 13, n 3, p. 151-153.

Hiernaux Jean, 1966, «Les Bushong et le Cwa du royaume Kuba (Congo Kinshasa) : Pygmées, Pygmoïdes et pygméisation; anthropologie, linguistique et expansion bantoue», Bulletin et mémoires de la Société d'anthropologie de Paris, vol. 9, n 11, p. 299-336.

Hombert Jean-Marie, Philippson Gérard et Demolin Didier, 2010, Linguistic Groupings of Hunters-Gatherers, manuscrit non publié.

Hulstaert Gustaaf, 1948, «Le dialecte des Pygmoïdes Batswa de l'Équateur», Africa : Journal of the International African Institute, vol. 18, n ${ }^{\circ}$ 1, p. 21-28.

Hulstaert Gustaaf, 1953, «Que signifie le nom Batswa?», Annales Aequatoria, vol. 16, p. 101-104.

Hulstaert Gustaaf, 1978, «Notes sur la langue des Bafoto», Anthropos, vol. 73, p. 113-133.

Hulstaert Gustaaf, 1986, «La langue des Jofe», Annales Aequatoria, vol. 7, p. 227-264.

Hulstaert Gustaaf, 1993, «Liste et carte des dialectes mongo», Annales Aequatoria, vol. 14, p. 401-406.

Hutereau Armand, 1909, «Notes sur la vie familiale et juridique de quelques populations du Congo belge», Annales d'ethnographie et anthropologie, Série III, Bruxelles, Musée du Congo belge.

Johnston Harry Hamilton, 1908, George Grenfell and the Congo, 2 vol., Londres, Hutchinson.

Molin S., 1933, «Notes sur les Boyela», Congo : revue générale de la colonie belge, vol. 14, p. 16-47.

Motingea Mangulu, 1994, «Notes sur les parlers des Pygmées d'Itendo (Zone de Kiri/Maindombe)», Annales Aequatoria, vol. 15, p. 341-381.

MulLER Ernst Wilhelm, 1964, «Die Batwa. Eine kleinwiichsige Jâgerkaste bei den Mongo-Ekonda» (Les Batwa. Une caste de chasseurs de petite taille chez les Mongo-Ekonda), Zeitschrift für Ethnologie, vol. 89, p. 206-215. 
Musée royal de l'Afrique centrale de Tervuren, <www.africamuseum.be/ museum/research/human-sciences/linguistics/lexico-1> (consulté le 3 mars 2013).

Rombauts Hugo, 1971, Batwá, Batúa, Batóa, Kinshasa, C.EP.

SeItz Stefan, 1997, «Carte 4 : Batwa des Mongo», dans S. Seitz, Pygmées d'Afrique centrale, Paris, Peeters / Selaf 338, p. 62.

Seitz Stefan, 1997, «Carte 5: Bacwa des Bakuba», dans S. Seitz, Pygmées d'Afrique Centrale, Paris, Peeters/Selaf 338, p. 64.

SEITz Stefan, 1997, Pygmées d'Afrique centrale, Paris, Peeters / Selaf 338.

VAn DER Kerken Georges, 1944, L'ethnie mongo, Bruxelles, G. van Campenhout.

VANsina Jan, 1954, Les tribus Ba-kuba et leurs peuplades apparentées, Tervuren, série Annales du Musée royal du Congo belge, monographies ethnographiques, 1 - XIII.

\section{Annexes}

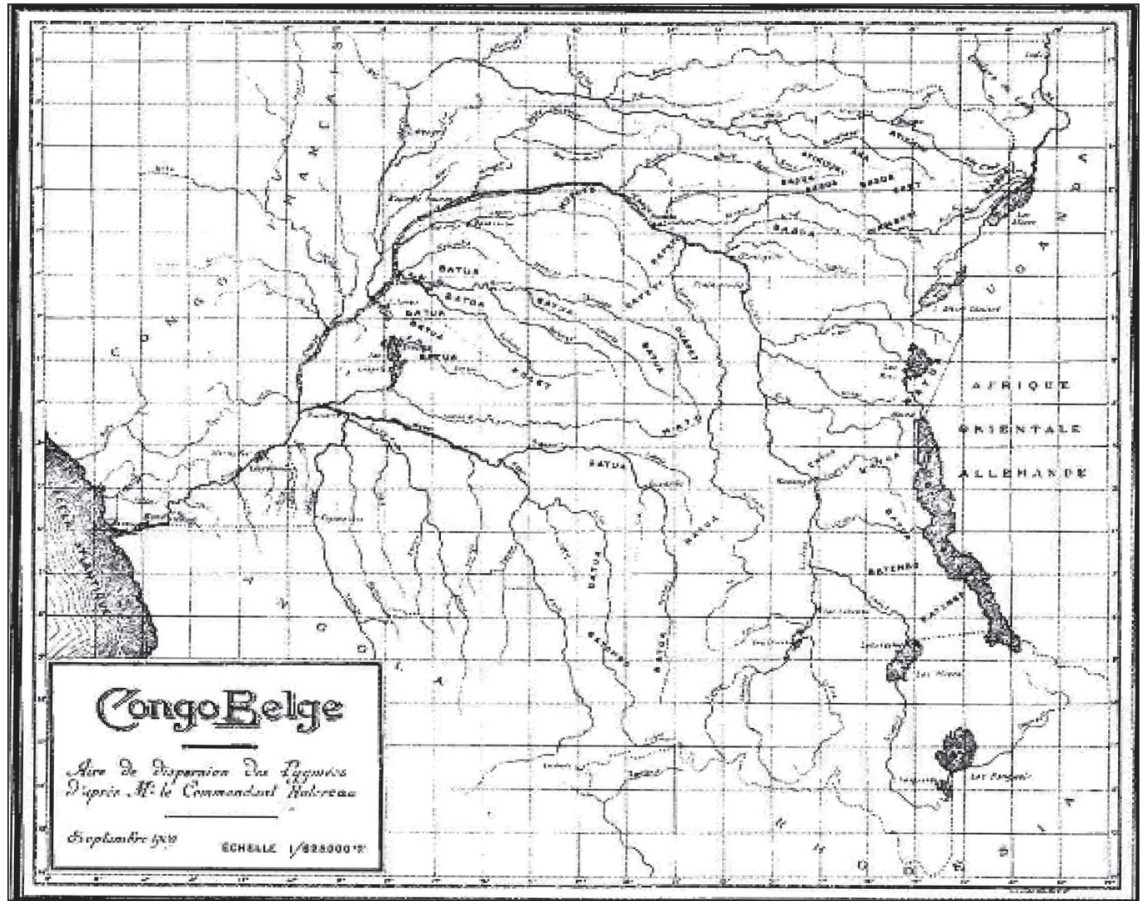

Carte du domaine des Pygmées au Congo Belge (Hutereau, 1909). 


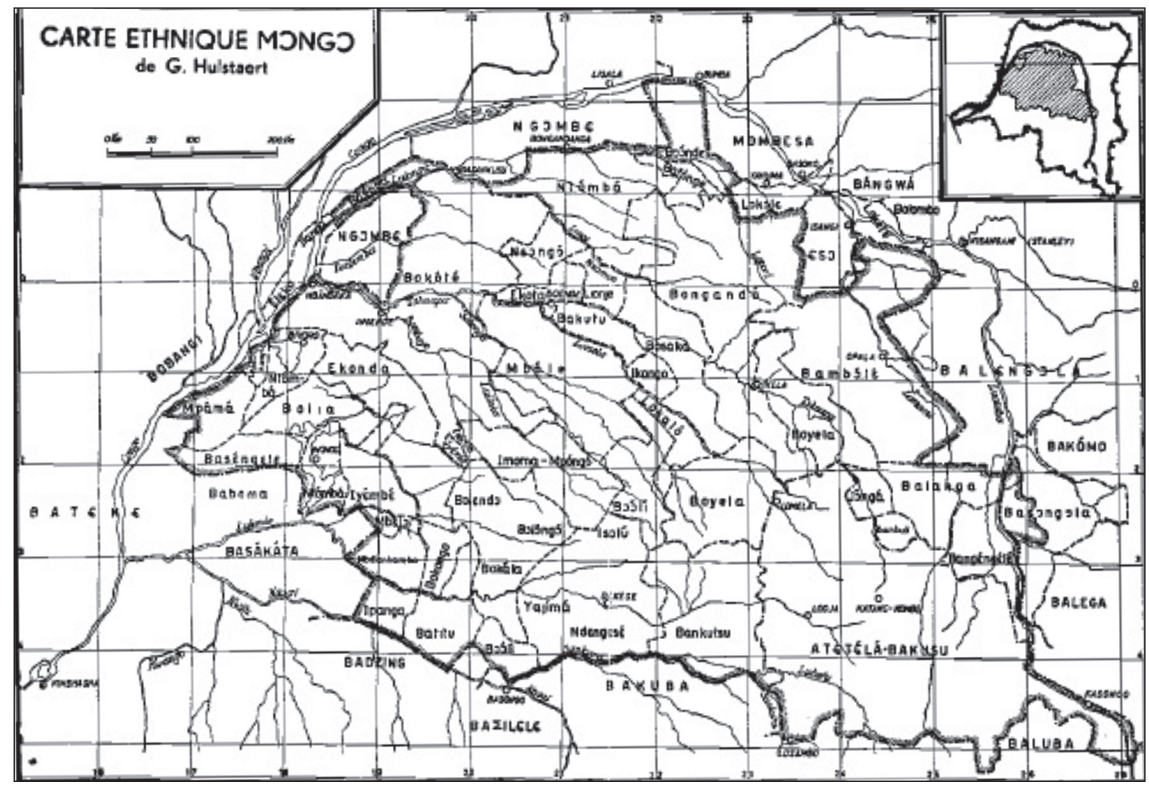

Carte ethnique Mongo (Hulstaert, 1993).

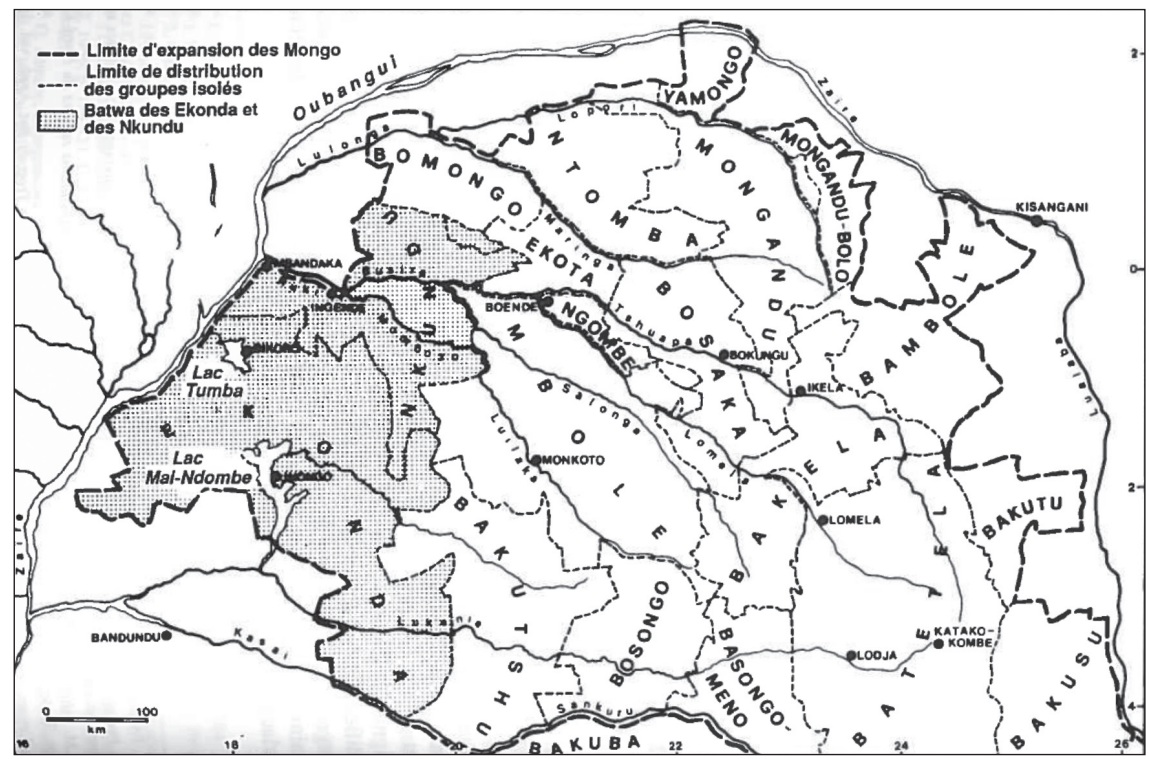

Localisation des Pygmées Batwa des Nkundo et des Ekonda (Seitz, 1997). 